\title{
On a Renormalization Group Approach to Dimensional Crossover
}

\author{
Ian Affleck ${ }^{1}$ and Bertrand I. Halperin ${ }^{2}$ \\ ${ }^{1}$ Canadian Institute for Advanced Research and Physics Department, University of British \\ Columbia, Vancouver, B.C., Canada, V6T1Z1 \\ ${ }^{2}$ Physics Department, Harvard University, Cambridge, MA02138, USA
}

(March 21, 2018)

\begin{abstract}
A recently proposed renormalization group approach to dimensional crossover in quasi-one-dimensional quantum antiferromagnets is improved and then shown to give identical results, in some cases, to those obtained earlier.
\end{abstract}

Recently, [1] a renormalization group (RG) approach was proposed to study a quasione dimensional quantum antiferromagnet, in which the ratio of inter-chain to intra-chain couplings, $R \rightarrow 0$. The approach works equally well for crossover to two or three dimensional behavior. It is based on a non-linear $\sigma$-model representation. In $\mathrm{D}$ space-time dimensions, using the imaginary time formalism, and rescaling time so that the spin-wave velocity is one, the action is written:

$$
S=\left(\Lambda^{D-2} / 2 g\right) \int d^{D} \vec{x}(\nabla \vec{\phi})^{2}
$$

Here $\vec{\phi}$ is the unit normalized n-component order parameter. It represents the threecomponent Néel order parameter for the Heisenberg antiferromagnet in (D-1) spatial dimensions at $T=0$. Alternatively, it could present a classical n-component magnetic order parameter in D spatial dimensions at finite temperature. $\Lambda$ is an ultraviolet cut-off and $g$ is the dimensionless coupling constant. In the spin-s quantum antiferromagnet, $g \approx 2 / s$ and increases with frustration. For the classical system, $g$ is proportional to the temperature, $T$.

There are actually two quite distinct cases, corresponding to integer or half-integer spin in the quantum system. For integer spin the $(1+1)$ dimensional system has a finite correlation 
length whereas for half-integer spin it is infinite (at $T=0)$, with power-law decay of the correlation function; i.e. quasi-long-range order. In the half-integer case, the $\sigma$-model action also contains a topological term, but this does not renormalize, so we need not keep track of it explicitly. [2] In this note we will focus on the half-integer case. Likewise a two-dimensional classical system has a finite correlation length (at finite temperature) for the Heisenberg case but an infinite correlation length in the xy case (for $T$ less than the Kosterlitz-Thouless temperature, $\left.T_{K T}\right)$. In the following paragraphs, we will use the language of classical two to three dimensional crossover. We then comment at the end on the implications for the quasi-one-dimensional quantum case and for various other dimensionalities.

In Ref. [四] the quasi-two dimensional case was represented by the same model, Eq. (11) after an appropriate rescaling of lengths in differenct directions, but with an ultraviolet cut-off which is much smaller in the weakly coupled (z) direction than in the two strongly coupled directions. Thus it was argued that one should use the two-dimensional RG until the cut-off for the strongly coupled directions is reduced to the same value as the other cut-off. Thereafter one should use the three-dimensional RG. Thus the first stage of renormalization just provides some effective bare coupling constant to be fed into in the three-dimensional RG equations.

The purpose of this note is to improve this argument in two ways. First of all, it is not really correct to use a continuum, derivative, representation for the weak inter-plane couplings in the intial stage of renormalization. Because the planes are weakly coupled the order parameters at adjacent points on neighboring planes are not neccessarily nearly parallel. Rather, we should use a discrete representation for this inter-plane coupling. Thus the appropriate action (or classical Hamiltonian) is

$$
\mathcal{S}=(1 / 2 g) \sum_{i} \int d^{2} \vec{r}\left[\left(\partial_{x} \vec{\phi}_{i}\right)^{2}+\left(\partial_{y} \vec{\phi}_{i}\right)^{2}+R \Lambda^{2} \vec{\phi}_{i} \cdot \vec{\phi}_{i+1}\right]
$$

Only in the second stage of renormalization can we use a gradient representation for the inter-plane coupling, as in Eq. (四). The other improvement is to take into account the rescaling of the field $\phi_{i}$ during the first stage of renormalization, 


$$
\vec{\phi} \rightarrow\left(\Lambda^{\prime} / \Lambda\right)^{x} \vec{\phi}
$$

Here $\Lambda^{\prime}<\Lambda$ is the reduced cut-off after the renormalization. $x$ is the scaling dimension of the field $\phi$. These improvements don't change the basic qualitative picture of Ref. [1] but lead to consistency with various earlier approaches, reviewed below.

To understand the neccessity for this rescaling and the significance of the exponent, $x$, consider a real-space block-spin RG transformation in two dimensions where the block has size $l$. That is we average $l^{2}$ unit-length spins. We assume that the spins in the block are approximately aligned due to the quasi-long-range order. However they do exhibit some random misalignment so that the averaged spin has a magnitude which is approximately

$$
\left|\vec{\phi}_{a v}\right| \approx l^{-x}
$$

The exponent $x$ can be seen to be the scaling dimension of the field. That is the correlation function goes as:

$$
<\vec{\phi}(\vec{r}) \cdot \vec{\phi}(\overrightarrow{0})>\propto r^{-2 x}
$$

We see this by letting the block size (possibly after many iterations) become $r$. At this stage, $\vec{r}$ and $\overrightarrow{0}$ are in the same block in the effective theory so they are aligned. The drop-off of the correlation function, from this view point arises from the reduction of the size of the block spin. In the momentum space renormalization group, discussed above and in Ref. [1], the block size is essentially $\Lambda / \Lambda^{\prime}$. Thus the averaged field appearing in the interplane coupling in Eq. (2) is $\left(\Lambda^{\prime} / \Lambda\right)^{x}$ times the unit-normalized field. Since we choose to work with a unit field in the non-linear $\sigma$-model approach, we must rescale $\vec{\phi}$ as in Eq. (3).

We switch over to the 3D RG when the coefficient of $\vec{\phi}_{i} \cdot \vec{\phi}_{i+1}$ (written in terms of the renormalized field) obeys the condition:

$$
R\left(\Lambda^{\prime} / \Lambda\right)^{2 x} \Lambda^{2} / 2 g \approx \Lambda^{\prime 2} / 2 g\left(\Lambda^{\prime}\right)
$$

At this stage it is appropriate to replace the lattice coupling by a continuous version:

$$
\Lambda^{\prime 2} \vec{\phi}_{i} \cdot \vec{\phi}_{i+1} \approx\left(\partial_{z} \vec{\phi}\right)^{2}
$$


The point is that now this coupling in the z-direction is about equally strong as that in the other directions, so the fields start to lock together in different planes. $\Lambda^{\prime}$ is the appropriate factor to adsorb into defining the derivative $\partial_{z}$ since it is the effective cut-off. Hereafter we use the 3D RG. Whether the system orders or not depends on whether the effective bare coupling for the 3D RG, obtained from the first stage of renormalization, is larger or smaller than the critical coupling $g_{c}$ in the 3D theory. In the two-dimensional theory, the coupling flows, in the infrared, to a limiting value $g_{2}(0)$. (See below.) Thus whether or not order occurs for infinitesimal $\mathrm{R}$ depends on whether $g_{2}(0)$ is larger or smaller than $g_{c}$. Since no general argument was apparent for which is larger, and it may not be universal, the question was investigated numerically in Ref. [1] for the square lattice $s=1 / 2$ antiferromaget. The conclusion was that $g_{2}(0)<g_{c}$, so order occurs for infinitesimal interplane coupling.

The above improvements change the previous analysis of Ref. [1] in two ways. First of all they change the estimate of the scale at which we switch to the higher dimensional RG. This scale, $\Lambda^{\prime}$ is now given by the above condition, Eq. (4), that is

$$
\Lambda^{\prime}=\Lambda R^{1 /[2(1-x)]}
$$

(We have assumed that $g / g\left(\Lambda^{\prime}\right) \approx g / g_{2}(0)$ is of order one. This assumption is discussed below.) This doesn't change the qualitative picture. The other important change involves the size of the ordered moment in the 3D phase. Since the bare coupling in the 3D theory, determined at the scale $\Lambda^{\prime}$, is of $O(1)$, so is the expectation value of the unrenormalized field $\vec{\phi}$, in the 3D theory. However, this unrenormalized field in the 3D theory has actually been rescaled by a factor of

$$
\left(\Lambda^{\prime} / \Lambda\right)^{x}=R^{x /[2(1-x)]}
$$

Therefore the actual magnetisation has a value:

$$
<\phi>\propto R^{x /[2(1-x)]}
$$

In Ref. [1] the "chain mean field theory" of [3] was generalized to deal with this type of crossover. In this approach the two dimensional system is treated exactly (in principle) but 
the higher dimensional coupling is treated in mean field theory. The mean field is

$$
h_{M F} \propto R<\phi>.
$$

In the lower dimensional $(\mathrm{D}=2)$ theory the magnetisation scales as:

$$
<\phi>\propto h_{M F}^{x /(2-x)} \propto(R<\phi>)^{x /(2-x)} .
$$

This gives:

$$
<\phi>\propto R^{x /[2(1-x)]}
$$

the same equation as obtained by the RG approach.

This "chain mean field theory" is also essentially equivalent to the crossover scaling theory of [4,5] as applied to the classical xy model in [6,7]. Now $g \propto T$. The two-dimensional theory exhibits a critical line, $g<g_{K T}$. Assuming that the Kosterlitz-Thouless temperature $g_{K T}<$ $g_{c}$, the three dimensional critical temperature, as is indicated by numerical simulations, the system orders for arbitrarily weak inter-plane coupling. The scaling exponent obeys $0<x<1 / 8$ along the critical line, reaching the value $1 / 8$ at $g_{K T}$.

For the half-integer spin Heisenberg quantum antiferromagnet, the $(1+1)$-dimensional coupling flows to a marginally stable fixed point, $g_{2}(0)$ separating the quasi-long-range ordered phase from the spontaneously dimerized phase, with $x=1 / 2$. (The $\mathrm{s}=1 / 2 \mathrm{xxz}$ chain with $\left|J^{z}\right|<J^{x}$, is similar to the classical xy model at $T<T_{K T}$, except that the exponent $x$ falls in the range $0<x<1 / 2$. [2]) The above analysis gives $<\phi>\propto R^{1 / 2}$ for the Heisenberg model. The ordered moment scales to zero as the square root of the inter-chain coupling.

In deriving Eq. (5) we have assumed that $g / g\left(\Lambda^{\prime}\right)$ is of order one. Generally, $g\left(\Lambda^{\prime}\right) \approx g_{2}(0)$ is $O(1)$ and the bare coupling, $g$, is also $O(1)$ so this assumption is valid. However, there are certain cases where $g<<1$. This occurs for a large-s antiferromagnet, where $g \approx 2 / s$. (It may also occur even for small $s$ with certain longer-range interactions that suppress quantum fluctuations.) Since the two-dimensional $\beta$-function is quadratic at small $g$,

$$
d g / d \ln \Lambda^{\prime} \approx-g^{2} / 2 \pi
$$


$g\left(\Lambda^{\prime}\right)$ remains small until the cutoff, $\Lambda^{\prime}$ has been reduced to exponentially small values, of $O\left(e^{-2 \pi / g}\right)$. Thus, assuming $R$, although small compared to 1 , is not exponentially small, that is,

$$
e^{-2 \pi / g}<<R<<1,
$$

we may essentially ignore the renormalization of $g$. The behavior of the two dimensional system is governed by the unstable $g=0$ fixed point which corresponds to long range order. We may still apply the above analysis, except that now $g\left(\Lambda^{\prime}\right)$ in Eq. (四) does not equal $g_{2}(0)$, but is rather approximately equal to $g$ and the effective value of $x$ is 0 . Therefore, from Eq. (6) the magnetisation is $\mathrm{O}(1)$ and from Eq. (5), the crossover scale is $\propto \sqrt{R}$. In this case "crossover" may not be the correct term since the system behaves as if it is ordered in both two-dimensional and three-dimensional regimes. $\sqrt{R}$ is simply the scale at which the three-dimensional dispersion becomes significant in a classical spin-wave analysis.

The above discussion assumed crossover from $(1+1)$ dimensions to $(2+1)$ dimensions, and numerical results on this case were presented in Ref. [1]. However, the above argument works equally well for crossover from $(1+1)$ to $(3+1)$ dimensions. This follows since the only property of the higher dimensional theory that was used was the fact that there is an order-disorder transition at finite $g$. Thus our analysis is relevant to real experimental systems such as $\mathrm{Sr}_{2} \mathrm{CuO}_{3}$. The crossover scale is, from Eq. (5), proportional to $R$. This means, for example, that the neutron scattering cross-section should exhibit one-dimensional behavior down to wave-vectors $k-\pi a \propto R$, where crossover to three-dimensional (Néel ordered) behavior should occur. A similar statement holds for the frequency or temperature dependence with the crossover (Néel) temperature being of order the inter-chain coupling. Crossover from $(2+1)$ to $(3+1)$ dimensions in quantum antiferromagnets was analysed by Chakravarty et al. [8]

To conclude, the RG approach of [1] to dimensional crossover suggests that order occurs for infinitesimal higher dimensional coupling only if the renormalized coupling in the lower dimensional theory, $g_{2}(0)$ is smaller than the critical coupling in the higher dimensional 
theory, $g_{c}$. In that case, this approach gives the same results as the mean field approach, or previous crossover scaling arguments. While all examples studied so far seem to obey $g_{2}(0)<g_{c}$, the other possibility may be realised in quasi-one-dimensional antiferromagnets with sufficiently frustrating inter-chain couplings.

\section{ACKNOWLEDGMENTS}

I.A. would like to thank K. Kojima and Y.J. Uemura for an interesting discussion. The research of I.A. was supported in part by NSERC of Canada and that of B.I.H. by N.S.F. Grant No. DMR94-16910. 


\section{REFERENCES}

1 I. Affleck, M.P. Gelfand and R.R.P. Singh, J. Phys. A27, 7313 (1994); Erratum 28, 1787 (1995).

2 For a review see I. Affleck, Fields, Strings and Critical Phenomena, ed. E. Brézin and J. Zinn-Justin, 563 [North-Holland, Amsterdam, 1990].

3 D. Scalapino, J. Imry and P. Pincus, Phys. Rev. B11, 2042 (1975).

4 R. Abe, Prog. Theor. Phys. 44, 339 (1970).

5 L.L. Liu and H.E. Stanley, Phys. Rev. Lett. 29, 927 (1972).

6 J.M. Kosterlitz and D.J. Thouless, Progress in Low Temperature Physics, vol 7B, [ed. D.F. Brewer, North-Holland, Amsterdam, 1978].

7 S. Hikami and T. Tsuneto, Prog. Theor. Phys. 63, 387 (1980).

8 S. Chakravarty, B.I. Halperin and D.R. Nelson, Phys. Rev. B39, 2344 (1989). 